\title{
Laser capture microdissection tailored to type 1 diabetes mellitus research
}

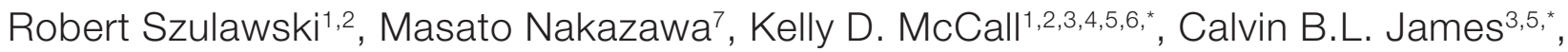 \\ and Frank L. Schwartz ${ }^{1,2,6, *}$ \\ ${ }^{1}$ Department of Specialty Medicine, ${ }^{2}$ Diabetes Institute, and ${ }^{3}$ Department of Biomedical Sciences, Ohio \\ University Heritage College of Osteopathic Medicine, Athens, $\mathrm{OH},{ }^{4}$ Department of Biological Sciences and \\ ${ }^{5}$ Molecular \& Cellular Biology Program, Ohio University College of Arts \& Sciences, Athens, OH ${ }^{6}$ Biomedical \\ Engineering Program, Ohio University Russ College of Engineering \& Technology Athens, $\mathrm{OH}$, and ${ }^{7}$ Office of \\ Research and Grants, Ohio University Heritage College of Osteopathic Medicine, Athens, $\mathrm{OH}$
}

*Denotes shared senior authorship.

BioTechniques 60:293-298 (June 2016) doi 10.2144/000114426

Keywords: laser capture microdissection (LCM); type 1 diabetes mellitus (TIDM); non-obese diabetic mouse (NOD); RNA isolation; cresyl violet; cryostat; toll-like receptor (TLR)

Supplementary material for this article is available at www.BioTechniques.com/article/114426.

RNA isolation from pancreatic islets poses unique challenges. Here, we present a reproducible means of obtaining high-quality RNA from juvenile rodent islets in sufficient quantities for use in ex vivo expression studies. Tissue was extracted from female non-obese diabetic (NOD) toll-like receptor 3 (TLR3) ${ }^{+/+}$ and $(\text { TLR3) })^{-1-}$ mice in the pre-diabetic stage. Samples were frozen in liquid nitrogen, sectioned, fixed in a highly alcoholic solution, and stained with an alcoholic cresyl violet (CV) solution. Rehydration of the fixed sections was minimized. Islets were identified visually and isolated with the Leica LMD6000 laser capture microdissection (LCM) system to yield samples highly enriched in islet RNA. Real time qPCR was performed on the islet cDNA using probes for CXC chemokine ligand 10 (CXCL10), an inflammatory marker that plays a critical role in the pathogenesis of type 1 diabetes mellitus (TIDM). This method represents an improvement over currently described LCM techniques for rodent pancreatic islets and makes feasible expression studies using small amounts of starting tissue without the need for RNA pre-amplification. This has immediate implications for ongoing TIDM studies using the NOD mouse.

The female non-obese diabetic (NOD) mouse is a widely studied and validated mammalian model of type 1 diabetes mellitus (TIDM). Our current understanding of the pathogenesis of TIDM in this animal model indicates that dendritic cells and macrophages, followed by $\mathrm{T}$ cells, are first observable in and around pancreatic islets at $\sim 4$ weeks of age $(1,2)$. After sufficient $T$ cell-mediated destruction of $\beta$-cell mass occurs, the onset of clinically apparent diabetes results, and $60 \%-100 \%$ of female NOD mice spontaneously develop overt diabetes by $4-6$ months of age $(3,4)$, depending on environmental factors and variability between mouse colonies (5). There are significant differences in the degree and nature of insulitis seen in the diabetic NOD mouse pancreas (diffuse and robust) compared with the insulitis in a new-onset TIDM patient (often, focal and modest) $(2,6)$. Impor- tantly, distribution of $\beta$ cells in the islet differs between rodents and humans (7). Nevertheless, there are major similarities between NOD and human TIDM, from the genetic linkage to specific human leukocyte antigen (HLA) subtypes and the contribution of major histocompatibility complex (MHC) class 1 overexpression to TIDM aggressiveness (1), to the milieu of chemokines and immune cells surrounding or invading the islets $(2,8)$.

\section{METHOD SUMMARY}

Here we introduce a method for isolating pancreatic islets for use in downstream expression studies on the pre-diabetic non-obese diabetic (NOD) mouse. Flash-frozen samples were fixed and stained with alcoholic cresyl violet solution and dehydrated in a manner that minimized RNase activity. RNA was isolated from stained islets by microdissection and used for multiplex real-time PCR. 
has shown involvement of the exocrine pancreas, with close association of CXCL10 with CD45-positive leukocytes outside the islets (21). Islet samples from non-diabetic healthy control patients show minimal CXCL10 expression as well as an absence of CXCR3-positive cell infiltration (21-23). CXCL10 expression in the vicinity of the islet is largely, but perhaps not exclusively, derived from $\beta$ cells and/or islet-infiltrating leukocytes in the animal model $(3,16,18,24)$ as well as in humans $(8,19)$. In summary, the pattern of islet CXCL10 expression and resulting CXCR3 positive T-cell infiltration in humans generally appears to be mirrored in the NOD model; however, the histopathology of infiltrating cells and resulting insulitis do differ in important ways, and insulitis is not necessarily present in at-risk individuals prior to the onset of TIDM, as it is in the pre-diabetic NOD mice (2).

\section{Materials and methods}

Female NOD/ShiLtJ mice (TLR3 ${ }^{+/+}$) were obtained commercially (\#001976; Jackson Laboratories, Bar Harbor, ME), and TLR3 knockout (TLR3 ${ }^{-/}$) mice (9) were kindly provided by Li Wen from Yale University. Following euthanasia, pancreata were removed, sectioned into pieces roughly $1 \mathrm{~cm}$ in the largest dimension, placed into Nunc cell culture tubes (Fisher Scientific, Pittsburg, PA), and flash-frozen in liquid nitrogen. Elapsed time from excision of the pancreas to freezing was $<5 \mathrm{~min}$. Molecular grade anhydrous ethanol and xylenes were used in all steps; excess reagent was placed in RNasefree sealed bottles with $3 \AA$ 4-8 mesh molecular sieves (\#208574; SigmaAldrich, St. Louis, MO), and the sealed containers were kept in a plastic tub filled with dessicant.

\section{Sectioning}

The CM1950 cryostat (Leica Biosystems, Buffalo Grove, IL) was defrosted, cleaned and treated with RNaseZap (Life Technologies, Grand Island, NY); Particular attention was given to treating the stage and anti-roll guide. Object temperature was set to $-18^{\circ} \mathrm{C}$, and the ambient cryostat temperature was set to $-20^{\circ} \mathrm{C}$. The blade (\#DT315R50; Sturkey, Lebanon, PA) was degreased and treated with RnaseZap and ethanol. A flat cold pack that had been removed from $-80^{\circ} \mathrm{C}$ was wrapped in RNaseZaptreated aluminum foil and placed in the cryostat immediately before sectioning. Samples were removed from liquid nitrogen and promptly affixed to the object disc of the cryostat with TissueTek Optimal Cutting Temperature (OCT) media (Sakura-Finetek USA, Torrence, CA). The affixed tissues were allowed to equilibrate with the cryostat environment for 10 min prior to sectioning.

$\beta$-cell width in another widely utilized rodent model, the Wistar rat, averages 10 microns in the largest dimension (25). One published protocol for LCM of rodent islets uses a section thickness of 14 microns (13), another protocol uses 8 micron-thick sections (14). In our experience, thicker sections had the desired effect of increasing the RNA yield for downstream processing. Therefore, pancreata were cut into sections 30-45 microns thick. The anti-roll guide was used to keep the sections flat against the stage as they were sectioned. Care was taken to avoid introducing any OCT to the section of interest, as OCT may reduce RNA yield during subsequent isolation steps (26) and can interfere downstream by inhibiting qPCR (12). If OCT is present, it must be removed prior to microdissection by aqueous rinses.

Polyethylene naphthalate membrane (PEN)-coated slides (\#LCM0522; Life Technologies) that had been previously treated with RNaseZap were used. Slides were rinsed twice in DEPC-treated water and placed under UV light for $4 \mathrm{~h}$ before use (Ohio State University. Laser capture microdissection protocols, slide preparation. https://lcm.osu.edu/lcma/ protocols/slide/index.cfm). To minimize RNA degradation, the slides were cooled to $0^{\circ} \mathrm{C}$ prior to contacting the samples in an RNase-free Coplin jar that was covered and partially submerged in an ice bath (27). A Kimwipe (\#34120; Kimtech Science, Roswell, GA) previously twisted to a point and placed in the cryostat, was used to manipulate the tissue section to preserve the orientation of serial sections (Claude Besmond, personal communication). Slides were quickly pressed parallel to the surface of the stage, and 6-10 serial sections adhered rapidly via the $20^{\circ} \mathrm{C}$ temperature gradient between the samples and the slides. A gloved finger was placed very briefly underneath the sections to ensure complete section contact with the slide. It is important not to completely melt the samples, as this will compromise morphology and RNA integrity. Immediately thereafter, the slides were placed membrane-side up on the wrapped cold pack to ensure rapid cooling of the sectioned samples. Handling of the slide was minimized to avoid heat transfer. As many as four unique samples were processed in one cutting session. Ethanol was used to clean the stage and anti-roll guide after each sample had been sectioned. Once sectioning was complete, and all 4 slides had cooled to $-80^{\circ} \mathrm{C}$ by contact with the cold pack, slides were rapidly transferred to an RNaseZap-treated Coplin jar that had been allowed to equilibrate with the cryostat. The jar was closed inside the cryostat, immediately placed in a cooled container filled with desiccant, and then transferred to $-80^{\circ} \mathrm{C}$ storage.

\section{RIN: 7.20}

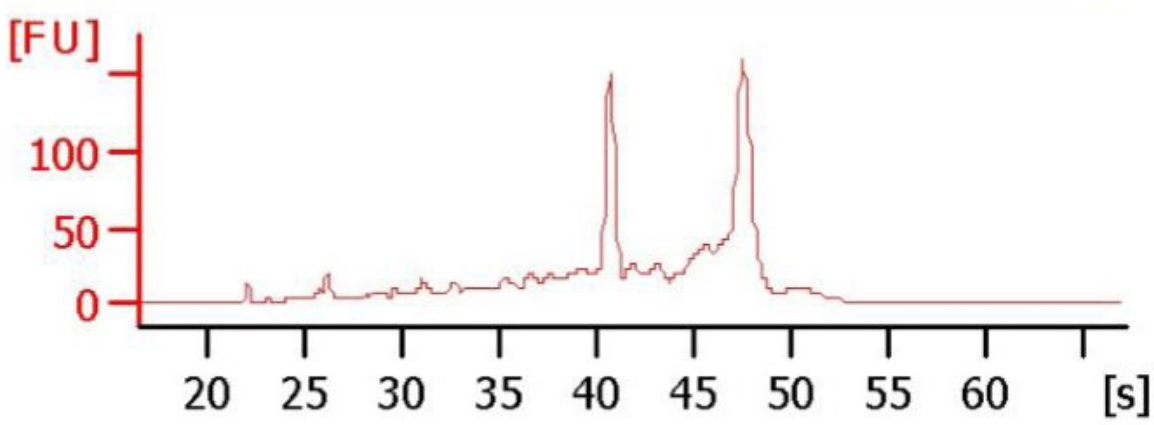

Figure 1. High quality RNA extracted from islets. Representative Bioanalyzer electropherogram of high-quality RNA extracted from microdissected islet using the described methodology. 
Table 1. Statistical models of the three outcomes as a function of week, genotype, and their interaction.

\begin{tabular}{|c|c|c|c|}
\hline & \multicolumn{3}{|c|}{ Outcome variables } \\
\hline & Mean $C_{q}(C X C L 10)$ & Mean $\mathrm{C}_{\mathrm{q}}$ (GAPDH) & Mean $\Delta \mathrm{C}_{q}(\mathrm{GAPDH}-\mathrm{CXCL} 10)$ \\
\hline \multicolumn{4}{|l|}{ Fixed effects } \\
\hline 1. Intercept (at week 5) & $31.67(2.09)^{* * *}$ & $24.94(1.25)^{* * *}$ & $-6.66(1.59)^{* * *}$ \\
\hline 2. Week & $-1.86(0.75)^{*}$ & $0.52(0.50)$ & $2.28(0.56)^{* * *}$ \\
\hline 3. Genotype & $-3.24(8.19)$ & $6.39(5.63)$ & $10.62(6.19)$ \\
\hline 4. Week $\times$ Genotype & $0.47(1.11)$ & $-1.07(0.83)$ & $-1.71(0.84)$ \\
\hline
\end{tabular}

Fixation and staining

Cresyl violet (CV) is a nucleophilic stain that has been shown to reliably stain pancreatic sections, allowing the visual distinction of islets from surrounding exocrine stroma (13), and our preliminary experiments found $\mathrm{CV}$ to give satisfactory morphological results. CV does not degrade RNA, making it an ideal stain for pancreatic sections $(27,28)$. In addition, highly alcoholic staining protocols have been shown to be superior to aqueous staining for maintaining RNA integrity, as rehydration allows nucleases present in the dehydrated tissue sections to work more efficiently and to degrade RNA more quickly (28-30). This observation is intuitive, as RNase activity requires free water (31), both as a cofactor for hydrolysis of nucleic acid and for stabilizing the enzyme in its active conformation (32). Slides were removed from $-80^{\circ} \mathrm{C}$ within 2 days of sectioning (12) and immediately fixed in 95\% ethanol for $30 \mathrm{~s}$ without defrosting. Working strength staining solution (see protocol in Supplementary Material) was added to the slide for 20-30 s.

\section{Dehydration}

Immediately after staining, the slides were transferred to two 95\% ethanol solutions for 1 min each, followed by 100\% ethanol, 2 times for 1 min each. Anhydrous ethanol was poured fresh for each use to minimize rehydration. Next, dehydrated samples were placed into a glass dish with anhydrous xylene (2 washes, 5 min each). Slides were then left to dry under the fume hood for 4-6 min to allow for evaporation of residual xylene before transfer to the previously cleaned LMD6000 stage for microdissection. Working time for LCM was kept to $<50 \mathrm{~min}$ in order to minimize rehydration of the sections from the ambient air (28).

\section{Laser capture microdissection}

The total number of TLR3\% samples processed was 18, while the total number of $\mathrm{TLR}^{+/+}$samples was 13 (Supplementary Table S3). Multiple sections of pancreas (head, middle, tail) were analyzed from several mice (total mice: $n=25$, total sampled sections: $n=31$ ). The proportion of $\beta$ cells as a percentage of total islet cells varies somewhat by region of pancreas (7); however, to our knowledge, studies do not exist that show a difference in insulitis burden between sections, and our data analysis accounted for any potential error this may have introduced, with section type evaluated as a variable effect in our mixed model analysis (results not shown). The total islet area from each section was collected using laser pulses of varying intensity (110-128) using the included LMD6000 software. The entire islet was obtained, without regard to peri-insulitis present in some of the islets. A thin border was allowed around each islet to minimize thermal damage from the laser. Three to 20 islets were collected at random and pooled per unique RNA sample. Pooled islets were dissolved with gentle agitation by pipette in RLT lysis buffer with added $\beta$-mercapatoethanol, as described in the manufacturer's protocol for the RNeasy Micro Kit (\#74004; Qiagen, Valencia, CA), and kept at $-80^{\circ} \mathrm{C}$. RNA extraction was performed using the RNeasy Micro Kit, and the RNA concentration in nuclease-free water was measured using a NanoDrop 2000 spectrophotometer (Thermo Scientific, Wilmington,
DE). Complementary strand synthesis with variable amounts of input RNA was immediately performed using the HighCapacity cDNA Reverse Transcription Kit (\#4368814; Life Technologies). Up to 4 samples were processed in 1 day.

\section{qPCR}

CXCL10 gene expression was detected using TaqMan Gene Expression Assay Mm00445235_m1 (Life Technologies). GAPDH was used as the housekeeping gene for all samples. As a quality measure, a subset of cDNA samples were aliquoted and evaluated for $18 \mathrm{~S}$ ribosomal RNA (Rn18S) as a second housekeeping gene. GAPDH and Rn18S are widely used as housekeeping genes as they are stably expressed in most tissues regardless of the experimental conditions, with exceptions that did not apply to our analysis $(12,14,33)$. The TaqMan Gene Expression Assays Mm99999915_g1 and Mm03928990_ g1 were used to detect the respective housekeeping genes. TaqMan Gene Expression Master Mix (\#4369016; Life Technologies ) was used with the Step-One-Plus Real-Time PCR System (Life Technologies). Assay primers were confirmed by the manufacturer to have log-linear expression levels throughout the entire detection range. Housekeeping genes reliably amplified in every well with $25 \mathrm{ng}$ input islet cDNA, run in duplicate or triplicate when possible, with a minimum total reaction volume of $50 \mu \mathrm{L}$. Good duplicate sample reliability was achieved with all reactions (CXCL10 Reporter dye: VIC, Cat \#4448490, Housekeeping Reporter dye, FAM, Cat \#4331182; both are part of the TaqMan Gene Expression Assay).

\section{Results and discussion}

The total RNA isolated from unique samples averaged $245 \mathrm{ng}$ per usable sample, a quantity sufficient to run studies on multiple genes without pre-amplification (11). Samples were occasionally lost due to handling

Table 2. The residual standard deviation (SD) of the three outcome variables at week 8 showing the change in the residual SD as a percentage of the SDS averaged over weeks 5-7.

\begin{tabular}{|l|l|l|l|}
\hline & \multicolumn{3}{|c|}{ Outcome variables } \\
\hline & Mean $\mathbf{C}_{\mathbf{q}}$ (CXCL10) & Mean $\mathbf{C}_{\mathrm{q}}$ (GAPDH) & $\Delta_{\mathrm{q}}$ (GAPDH - CXCL10) \\
\hline SD at week 8 & 0.82 & $0.42^{* *}$ \\
\hline$\%$ of weeks 5-7 average & $0.39 *$ & \\
\hline$* * * P<0.001,{ }^{*} P<0.01,{ }^{*} P<0.05$.
\end{tabular}


errors or PEN membrane slide failure, including poor adhesion of samples. A usable quantity of RNA, defined as at least $25 \mathrm{ng}$ of RNA available for cDNA synthesis, was isolated from as few as 3 pancreatic islets with a total surface area of $44,535 \mu \mathrm{m}^{2}$, while the average yield of RNA was 22 ng per 100,000 $\mu \mathrm{m}^{2}$. Areas were measured as the laser lasso was drawn around the islet to be collected. Supplementary Figures S1 and S2 show an islet before and during collection. A subset of previously frozen RNA samples were run using a RNA 6000 Pico kit on the 2100 Bioanalyzer system (Agilent Technologies, Santa Clara, CA). RIN values (34) as high as 7.2 were obtained (Figure 1).

Real-time PCR data were analyzed using mixed effects modeling. The TLR3 ${ }^{-/}$ genotype was used as a reference for fixed effects. As predicted, $\log _{10}$-transformed CXCL10 [ $\left.\log _{10}(\mathrm{CXCL10})\right]$ decreased from 5 to 8 weeks $(P<0.05$ Table 1, Model 1), as indicated by a lower threshold cycle $\left(\mathrm{C}_{q}\right)$ value, and thus higher CXCL10 expression. Neither the baseline $\log _{10}(\mathrm{CXCL10})$ level nor the rate of change over time differed between the TLR3 ${ }^{+/+}$and TLR3 $3^{-/}$animals (p's > 0.2). No significant difference in GAPDH expression between the two groups and by age was apparent (p's $>0.2$ ) (Table 1, Model 2).

$\log _{10}$-transformed $\Delta \mathrm{C}_{\mathrm{q}}(\mathrm{GAPDH}$ CXCL10) $\left[\log _{10}\left(\Delta C_{q}\right)\right]$ values increased with age ( $P<0.001$, Table 1, Model 3), indicating a rise in CXCL10 expression as the mice matured. There was not diffrence between the genotypes either in the baseline $\log _{10}\left(\Delta C_{q}\right)$ level at 5 weeks $(P>0.2)$ or in the rate of change over time $(P>0.2)$, indicating no effect of genotype on average CXCL10 expression. These results were as predicted, based on our previous research that has shown no difference in the incidence of diabetes in uninfected TLR3 ${ }^{-/-}$vs TLR3 ${ }^{+/+}$NOD mice (10). Observations were plotted, with model best-fit regression line in blue, with the gray shading representing the 95\% confidence interval (Supplementary Figure S3).

The residual standard deviations (SDs) of $\log _{10}(C X C L 10)$ and $\log _{10}\left(\Delta C_{q}\right)$ at week 8 for both genotypes were lower than the average of the previous weeks by $60 \%$ (both $P$ values $<0.05$ ). The amount of reduction in the SD did not differ between the TLR3 ${ }^{-/-}$and TLR3 ${ }^{+/+}$ mice $(P>0.2)$ (Table 2$)$. These results were expected, owing to the variable nature of the onset and progression of insulitis in the NOD mouse (2). In our experience, young mice from the same cohort may have differing levels of insulitis when considered at a particular time point, reaching more consistent levels of insulitis with maturity.

The data presented here show no difference in the average values of a crucial marker of insulitis between $\mathrm{TLR}^{+/+}$and $\mathrm{TLR}^{-/} \mathrm{NOD}$ mice, from ages 5 to 8 weeks, but does show an increase in the expression of CXCL10 with age in both genotypes. A strong correlation between $\Delta \mathrm{C}_{\mathrm{q}}(\mathrm{GAPDH}-$ CXCL10) and $\Delta C_{q}(R n 18 S-C X C L 10)$ qPCR values run on separate aliquots of islet cDNA samples $(n=19)$ was observed (Pearson's $r=0.78 \mathrm{df}=17$, $P<0.01)$, confirming that islet cDNA was of adequate quality for performing expression studies.

Our aim was to reduce some of the difficulty inherent to performing expression studies on islets. The results reinforce the importance of minimizing rehydration of frozen tissues containing elevated RNase levels, as well as choosing appropriate fixing, staining, and dehydration techniques to minimize degradation of ribonucleic acids in such challenging tissues. We have reproducibly isolated sufficient quantities of islet RNA for an expression study, without the need for pre-amplification. The use of costly reagents is minimized, and multiple samples can be processed in 1 day. RNA quality compares favorably to existing protocols, ensuring confidence in the interpretation of downstream PCR data.

There have been no previously published results of quantitative real time PCR multiplexed with endogenous controls on cDNA isolated from native islets in 5-8 week old NOD mice. The success of this technique will facilitate the use of the pre-diabetic animals for TIDM research. LCM gives the researcher a powerful tool to gain insight into the condition of the islet transcriptome at the moment the animal was sacrificed. Ex vivo data thus gathered may give insights into the influence of variables, both exogenous and endogenous to the patient, that play a role in the genesis of insulitis that is critical to the progression of TIDM in humans.

There is room for improvement of the method in several areas. Further work will address optimization of the freezing process for pancreas samples, maximizing RNA integrity while preserving morphology for visual identification of islets. Alternatives to liquid nitrogen, such as isopentane and dry ice, have not yet been explored by our team. While overall RNA quality was high, fluctuations in ambient humidity during the sectioning and staining process may undermine efforts to keep tissue dehydrated and will also be investigated to minimize variability between samples. Nevertheless, this work represents a substantial improvement in the application of LCM technology to pertinent issues in TIDM research and will augment the utility of the already valuable NOD model.

\section{Author contributions}

R.S., C.B.L.J., K.D.M., and F.L.S. contributed to the original idea for the study. R.S. conducted RNA isolation and qPCR studies and writing and editing of the manuscript. C.B.L.J., K.D.M., and F.L.S. helped guide the study and interpret results, and supervised all aspects of the work, including writing and editing of the manuscript. M.N. assisted with applied statistical modeling and presentation of qPCR data.

\section{Acknowledgments}

The authors would like to thank Claude Besmond at Necker-Sick Children Hospital for correspondence related to technical aspects of LCM, and cryostat and slide preparation; Tarik Attasi for data collection; Ashley Patton and Ladonya Jackson for discussions and assistance in executing qPCR; Ramiro Malgor for discussion related to technical aspects of LCM; and Jean Thuma for expertise in husbandry of the NOD mice. Animal studies were conducted with approval from the Ohio University Institutional Animal Care and Use Committee in accordance with accepted standards of humane animal care. This work was supported in part by the National Institutes of Health, NIDDK 
[grant 1R15 DK081192-01 to K.D.M.], the J.O. Watson Endowed Chair for Diabetes Research, and funds awarded to R.S. by the Ohio University Heritage College of Osteopathic Medicine Centers for Osteopathic Research and Education (CORE). This paper is subject to the NIH Public Access Policy.

\section{Competing interests}

The authors declare no competing interests.

\section{References}

1. Driver, J.P., D.V. Serreze, and Y.G. Chen. 2011. Mouse models for the study of autoimmune type 1 diabetes: a NOD to similarities and differences to human disease. Semin. Immunopathol. 33:67-87.

2. In't Veld, P. 2014. Insulitis in human type 1 diabetes: a comparison between patients and animal models. Semin. Immunopathol. 36:569-579.

3. Shigihara, T., Y. Oikawa, Y. Kanazawa, Y. Okubo, S. Narumi, T. Saruta, and A. Shimada. 2006. Significance of serum CXCL10/IP-10 level in type 1 diabetes. J. Autoimmun. 26:66-71.

4. McCall, K.D., M.J. Schmerr, J.R. Thuma, C.B. James, M.C. Courreges, F. Benencia, R. Malgor, and F.L. Schwartz. 2013. Phenylmethimazole suppresses dsRNA-induced cytotoxicity and inflammatory cytokines in murine pancreatic beta cells and blocks viral acceleration of type 1 diabetes in NOD mice. Molecules 18:3841-3858.

5. Pozzilli, P., A. Signore, A.J. Williams, and P.E. Beales. 1993. NOD mouse colonies around the world--recent facts and figures. Immunol. Today 14:193-196.

6. Korsgren, S., Y. Molin, K. Salmela, T. Lundgren, A. Melhus, and O. Korsgren. 2012. On the etiology of type 1 diabetes: a new animal model signifying a decisive role for bacteria eliciting an adverse innate immunity response. Am. J. Pathol. 181:1735-1748.

7. Suckale, J. and M. Solimena. 2008. Pancreas islets in metabolic signaling--focus on the beta-cell. Front. Biosci. 13:7156-7171.

8. Uno, S., A. Imagawa, K. Saisho, K. Okita, H. Iwahashi, T. Hanafusa, and I. Shimomura. 2010. Expression of chemokines, CXC chemokine ligand 10 (CXCL10) and CXCR3 in the inflamed islets of patients with recentonset autoimmune type 1 diabetes. Endocr. J. 57:991-996.

9. Wong, F.S., C. Hu, L. Zhang, W. Du, L. Alexopoulou, R.A. Flavell, and L. Wen. 2008. The role of Toll-like receptors 3 and 9 in the development of autoimmune diabetes in NOD mice. Ann. N. Y. Acad. Sci. 1150:146148.

10. McCall, K.D., J.R. Thuma, M.C. Courreges, F. Benencia, C.B. James, R. Malgor, N. Kantake, W. Mudd, et al. 2015. Toll-like receptor 3 is critical for coxsackievirus B4-induced type 1 diabetes in female NOD mice. Endocrinology 156:453-461.
11. Bynum, J.W. and R.A. Ronzio. 1976. An improved method for the rapid isolation of RNA from tissue with high ribonuclease content. Anal. Biochem. 73:209-214.

12. Erickson, H.S., P.S. Albert, J.W. Gillespie, J. Rodriguez-Canales, W. Marston Linehan, P.A. Pinto, R.F. Chuaqui, and M.R. Emmert-Buck. 2009. Quantitative RT-PCR gene expression analysis of laser microdissected tissue samples. Nat. Protoc. 4:902-922.

13. Dreja, T., Z. Jovanovic, A. Rasche, R. Kluge, R. Herwig, Y.C. Tung, H.G. Joost, G.S. Yeo, and H. Al-Hasani. 2010. Dietinduced gene expression of isolated pancreatic islets from a polygenic mouse model of the metabolic syndrome. Diabetologia 53:309-320.

14. Shan, Z., B. Xu, A. Mikulowska-Mennis, and S.A. Michie. 2014. CCR7 directs the recruitment of $T$ cells into inflamed pancreatic islets of nonobese diabetic (NOD) mice. Immunol. Res. 58:351-357.

15. Vermeulen, J., S. Derveaux, S. Lefever, E. De Smet, K. De Preter, N. Yigit, A. De Paepe, F. Pattyn, et al. 2009. RNA pre-amplification enables large-scale RT-qPCR gene-expression studies on limiting sample amounts. BMC Res Notes. 2:235.

16. Ahmadi, Z., M.K. Arababadi, and G. Hassanshahi. 2013. CXCL10 activities, biological structure, and source along with its significant role played in pathophysiology of type I diabetes mellitus. Inflammation 36:364-371.

17. Groom, J.R. and A.D. Luster. 2011. CXCR3 ligands: redundant, collaborative and antagonistic functions. Immunol. Cell Biol. 89:207215.

18. Frigerio, S., T. Junt, B. Lu, C. Gerard, U. Zumsteg, G.A. Hollander, and L. Piali. 2002. Beta cells are responsible for CXCR3mediated T-cell infiltration in insulitis. Nat. Med. 8:1414-1420.

19. Antonelli, A., S.M. Ferrari, A. Corrado, E. Ferrannini, and P. Fallahi. 2014. CXCR3, CXCL10 and type 1 diabetes. Cytokine Growth Factor Rev. 25:57-65.

20. Midgley, A., C. Thorbinson, and M.W. Beresford. 2012. Expression of Toll-like receptors and their detection of nuclear selfantigen leading to immune activation in JSLE. Rheumatology (Oxford) 51:824-832.

21. Sarkar, S.A., C.E. Lee, F. Victorino, T.T. Nguyen, J.A. Walters, A. Burrack, J. Eberlein, S.K. Hildemann, and D. Homann. 2012. Expression and regulation of chemokines in murine and human type 1 diabetes. Diabetes 61:436-446.

22. Tanaka, S., Y. Nishida, K. Aida, T. Maruyama, A. Shimada, M. Suzuki, H. Shimura, S. Takizawa, et al. 2009. Enterovirus infection, CXC chemokine ligand 10 (CXCL10), and CXCR3 circuit: a mechanism of accelerated beta-cell failure in fulminant type 1 diabetes. Diabetes 58:2285-2291.

23. Roep, B.O., F.S. Kleijwegt, A.G. van Halteren, V. Bonato, U. Boggi, F. Vendrame, P. Marchetti, and F. Dotta. 2010. Islet inflammation and CXCL10 in recent-onset type 1 diabetes. Clin. Exp. Immunol. 159:338-343.
24. Martin, A.P., M.G. Grisotto, C. CanastoChibuque, S.L. Kunkel, J.S. Bromberg, G.C. Furtado, and S.A. Lira. 2008. Islet expression of M3 uncovers a key role for chemokines in the development and recruitment of diabetogenic cells in NOD mice. Diabetes 57:387-394.

25. Zimny, M.L. and W.G. Blackard. 1975. The surface structure of isolated pancreatic islet cells. Cell Tissue Res. 164:467-471.

26. Cox, M.L., C.L. Schray, C.N. Luster, Z.S. Stewart, P.J. Korytko, K.N. Khan, J.D. Paulauskis, and R.W. Dunstan. 2006. Assessment of fixatives, fixation, and tissue processing on morphology and RNA integrity. Exp. Mol. Pathol. 80:183-191.

27. Clément-Ziza, M. 2007. Approche transcriptomique du systeme nerveux enterique et de la maladie de Hisrschsprung. Doctoral Thesis, University Paris Descartes, Paris, France.

28. Clément-Ziza, M., A. Munnich, S. Lyonnet, F. Jaubert, and C. Besmond. 2008. Stabilization of RNA during laser capture microdissection by performing experiments under argon atmosphere or using ethanol as a solvent in staining solutions. RNA 14:26982704.

29. Cummings, M., C.V. McGinley, N. Wilkinson, S.L. Field, S.R. Duffy, and N.M. Orsi. 2011. A robust RNA integrity-preserving staining protocol for laser capture microdissection of endometrial cancer tissue. Anal. Biochem. 416:123-125.

30. Ordway, G.A., A. Szebeni, M.M. Duffourc, S. Dessus-Babus, and K. Szebeni. 2009. Gene expression analyses of neurons, astrocytes, and oligodendrocytes isolated by laser capture microdissection from human brain: detrimental effects of laboratory humidity. J. Neurosci. Res. 87:2430-2438.

31. Fend, F., M.R. Emmert-Buck, R. Chuaqui, K. Cole, J. Lee, L.A. Liotta, and M. Raffeld. 1999. Immuno-LCM: laser capture microdissection of immunostained frozen sections for mRNA analysis. Am. J. Pathol. 154:61-66.

32. Wlodawer, A., R. Bott, and L. Sjolin. 1982. The refined crystal structure of ribonuclease A at 2.0 A resolution. J. Biol. Chem. 257:13251332.

33. Moreno-Igoa, M., R. Manzano, S. Olivan, A.C. Calvo, J.M. Toivonen, and R. Osta. 2010. Effects of gene therapy on muscle $18 \mathrm{~S}$ rRNA expression in mouse model of ALS. BMC Res Notes. 3:275.

34. Schroeder, A., O. Mueller, S. Stocker, R. Salowsky, M. Leiber, M. Gassmann, S. Lightfoot, W. Menzel, et al. 2006. The RIN: an RNA integrity number for assigning integrity values to RNA measurements. BMC Mol. Biol. 7:3.

Received 25 July 2015; accepted 03 March 2016.

Address correspondence to Kelly McCall, Ohio University Heritage College of Osteopathic Medicine, 302B Academic Research Center, Athens, $\mathrm{OH}$ 45701. E-mail: mccallk@ohio.edu

To purchase reprints of this article, contact: biotechniques@fosterprinting.com 